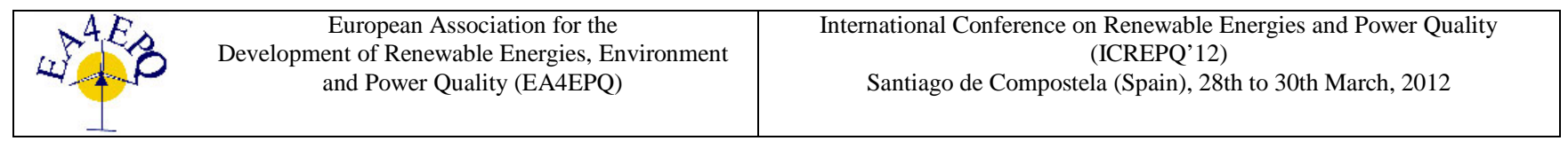

\title{
Frequency Stability Analysis of a Dynamic Wind Park in an Integrated Power Grid
}

\author{
L. Thurner ${ }^{1}$, S. Liu ${ }^{1}$ \\ ${ }^{1}$ Department of Electrical and Computer Engineering \\ University of Kaiserslautern \\ Erwin-Schrödinger-Str. 12, 67663 Kaiserslautern (Germany) \\ e-mail: leonthurner@posteo.de, sliu@eit.uni-kl.de
}

\begin{abstract}
In spite of rapidly growing wind power capacities, wind power still only accounted for a small fraction of the electric power supply in the last years. Therefore, research in the area of wind power was mostly focused on the wind generators and wind parks themselves, aiming to maximize their efficiency and stability. But as the weight of wind power grows, their influence on power systems cannot be neglected anymore. That is why recent research like [1], [2] or [3] studies the effects that wind parks have on the dynamic power grids they are connected to. Continuing in this direction, we implement a simulation model of a power grid including a wind park and study its dynamic behavior with varying wind speed. The influence of certain parameters like transmission line length, rated power of the wind park or control depth on the frequency stability in the power system are studied and analyzed. Furthermore, a control scheme to integrate the wind park into the grid frequency control is implemented.
\end{abstract}

\section{Key words}

Wind Park, Doubly-Fed Induction Generator (DFIG), Integrated Power Grid, Frequency Stability, Frequency Control

\section{Introduction}

Since the power generation of wind turbines depends on the fluctuating wind speed, wind parks can be seen as erratic power sources which can cause transient oscillations. These fluctuations in power input have to be balanced by the traditional power plants in the power grid. As long as the fraction of wind energy remains small, integrated power grids are able to absorb those perturbations. With the rising penetration of wind energy, however, the fluctuations grow larger and the effort that has to be invested to stabilize the power grid increases.

The issue of integrating renewable energy sources in integrated power grids has been addressed in recent research such as [1] or [2]. These papers make important contributions towards understanding voltage stability and reactive power supply during grid fault. In this paper, however, we will address the issue with a focus on active power supply and frequency stability, where connections and interrelations are not as well documented yet. First, we simulate different situations to better understand the behavior of the system and the influence of certain parameters. Based on these results we then present a control strategy that allows the wind park to participate in frequency control and help stabilize the power grid. The work in this part is based on research presented in [3] that presents a good solution to integrate wind generators into the grid frequency control. Since the solution is verified mostly qualitatively in [3], we study quantitative results to determine the efficiency of the proposed solution and propose an extension to improve performance.

The paper is divided into the following parts: first, the simulation models of the wind park (Chapter 2) and the power system (Chapter 3) that were implemented in Matlab/Simulink are presented. In Chapter 4, simulation results for the frequency stability are presented (Sections A to C) and a DFIG frequency control scheme is proposed and verified (Section D). The paper is closed by a conclusion in Chapter 5.

\section{Wind Park Model}

\section{A. Wind Turbine Model}

The drive drain and the generator rotor are modeled as a one mass system, which means that stiffness and damping effects are neglected. Therefore, the angular velocity $\omega_{\mathrm{r}}$ is the same for the generator rotor and the turbine rotor and can be calculated as:

$$
\dot{\omega}_{r}=\frac{T_{m}-T_{e}}{2\left(\mathrm{H}_{\mathrm{t}}+\mathrm{H}_{\mathrm{g}}\right)}
$$

with $\mathrm{H}_{\mathrm{t}}$ and $\mathrm{H}_{\mathrm{g}}$ being the turbine and generator inertia constants and $\mathrm{T}_{\mathrm{m}}$ and $\mathrm{T}_{\mathrm{e}}$ being the mechanical and electrical torque respectively. The mechanical torque $T_{m}$ results from the wind and therefore depends on the current wind speed. The wind speed profile used in our simulations is depicted in Figure 1.

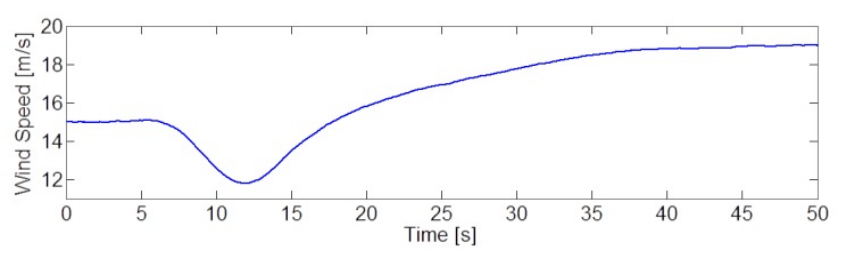


Fig. 1. Simulated Wind Profile

The electrical torque $T_{e}$ is provided by the electrical generator which is modeled as a doubly-fed induction generator (DFIG). In addition to the stator that is directly connected to the power grid, the rotor is connected to the power grid through a converter (see Figure 2). This kind of generator is widely used in wind turbines and common models are documented in the literature, such as [4] or [5].

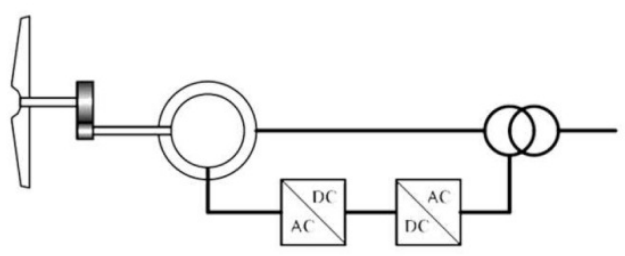

Fig. 2. Doubly-Fed Induction Generator [4]

We implement a first-order model of the DFIG, which means transients in rotor and stator flux are neglected. The voltages in rotor and stator are then given as:

$$
\begin{aligned}
& \underline{u}_{s}=-R_{s} \underline{i}_{s}+j \omega_{s}\left[\underline{i}_{r}\left(L_{s l}+L_{m}\right)-\underline{i}_{r} L_{m}\right] \\
& \underline{u}_{r}=-R_{r} \underline{i}_{r}+j \omega_{r}\left[\underline{i}_{s}\left(L_{r l}+L_{m}\right)-\underline{i}_{s} L_{m}\right]
\end{aligned}
$$

The subscripts $r$ and $s$ stand for rotor and stator respectively, and $\mathrm{L}_{l}$ and $\mathrm{L}_{\mathrm{m}}$ are the leakage and the mutual inductances respectively.

The rotor current $\underline{i}_{r}$ is used to control the behavior of the wind turbine by influencing the currents injected by the rotor side converter. By designing the control system in a dq-reference frame with stator flux orientation, active power generation and stator voltage can be controlled independently. The q-component of the rotor current is used to control the active power output according to a desired reference value $P_{e}^{\text {ref. }}$

$$
i_{q r}^{r e f}=\frac{P_{e}^{r e f}\left(L_{s l}+L_{m}\right) \omega_{s}}{\left|u_{s}\right| L_{m} \omega_{r}}
$$

Where $\omega_{s}$ is the synchronous angular velocity of the induction generator and $u_{s}$ is the voltage phasor at the stator.

The d-component of the rotor current is used to control the stator voltage to its rated value $U_{s}^{\text {ref }}$ :

$$
i_{d r}^{r e f}=\frac{Q_{s}\left(L_{s l}+L_{m}\right)}{\left|\underline{u}_{s}^{r e f}\right| L_{m}}-\frac{\left|\underline{u}_{s}^{r e f}\right|}{\omega_{s} L_{m}}
$$

Where $Q_{s}$ is the reactive power at the stator.

The model also includes a pitch angle controller that pitches the rotor blades at strong wind speeds and a protection system that disconnects the DFIG from the power grid when a fault occurs. The full control scheme can be seen in Figure 3.

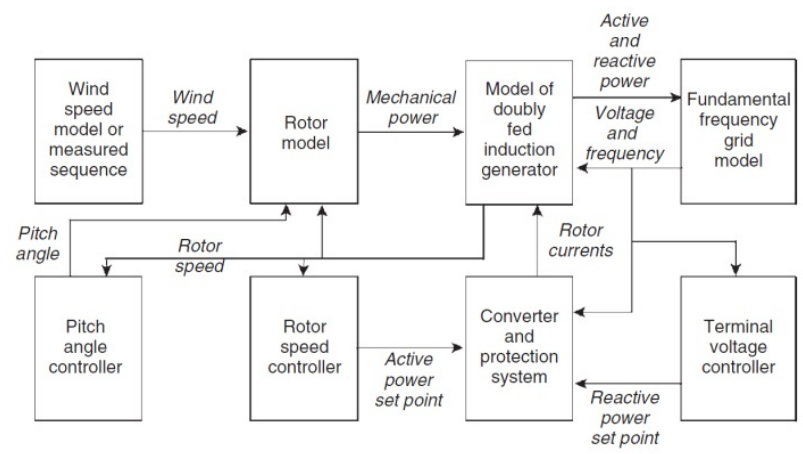

Fig. 3. DFIG Control Scheme [4]

\section{B. Electrical and Geographical Structure}

The wind park consists of six wind turbines, each with a rated stator voltage of $690 \mathrm{~V}$ and a rated power of 2 MVA (see Figure 4).

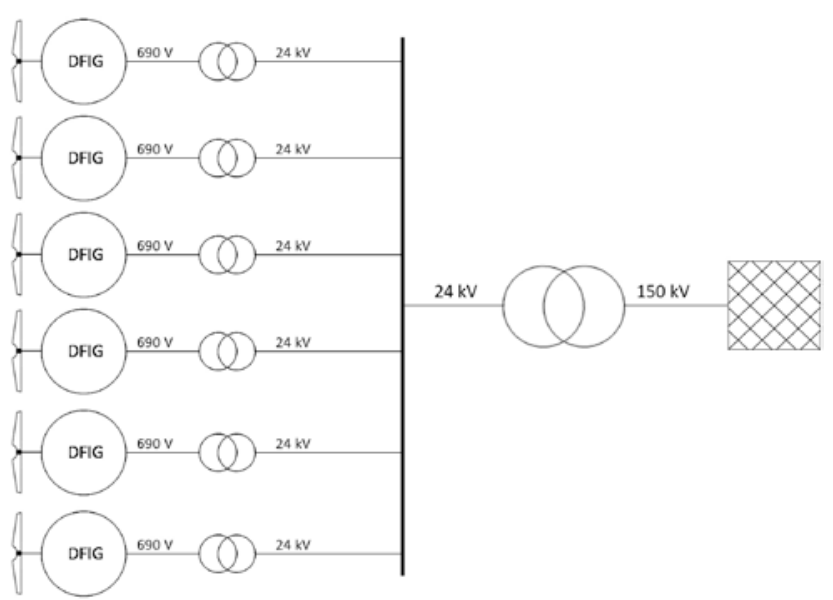

Fig. 4. Electrical Structure of the Wind Park

The stator voltage is transformed to $24 \mathrm{kV}$ and then connected at the point of common coupling (PCC) via transmission lines. After all lines are connected at the PCC bus, the voltage is transformed to the level of the power supply grid, which equals $150 \mathrm{kV}$.

The geographical structure of the wind park can be seen in Figure 5. It is accounted for in the model by including the wake that diminishes the effective wind speed when the wind passes the rotor blades of a wind turbine. The wind power input of wind turbines located in the wake cone of other wind turbines is diminished according to the Jensen-model [6]:

$$
v_{w, w a k e}=v_{w} \frac{1-\sqrt{1-C_{t}}}{\left(1+\frac{2 k x}{d_{0}}\right)^{2}}
$$

Where $v_{w}$ is the actual wind speed, $x$ is the distance between wind turbines, $k$ the slope of the wake cone, $C_{t}$ the thrust coefficient and $d_{0}$ the rotor diameter. The model is linear and two-dimensional which makes it easy to calculate. Since we are only interested in the electrical behavior of the wind park, this model is sufficiently accurate to guarantee individual electrical states for every wind turbine and represent the electrical dynamics in the wind park. 


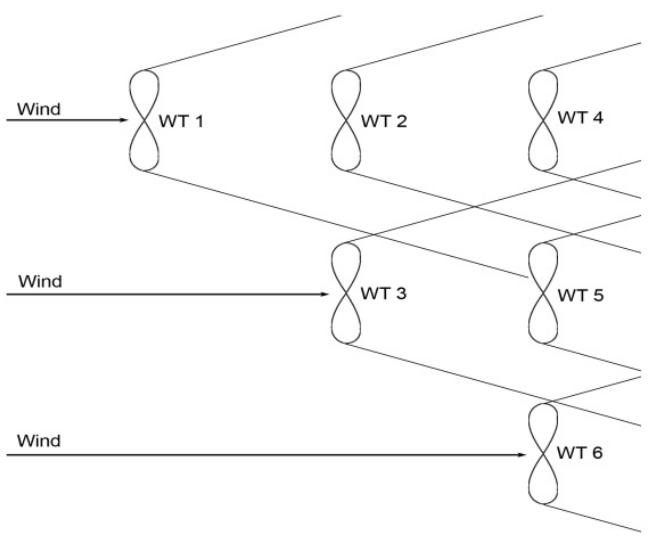

Fig. 5. Wind Park Topology

\section{Reactive Power Control}

The wind park is also equipped with a reactive power controller that allows the wind park to operate at a desired power factor. Individual wind turbines are usually controlled to compensate for their own reactive power need, but inductances in cables and transformers cause additional reactive power that has to be compensated. To operate the entire wind park with a desired power factor $\cos (\varphi)_{\text {ref }}$, the reactive power has to be controlled to:

$$
Q_{r e f}=P_{p c c} \sqrt{\frac{1}{\cos (\phi)_{r e f}^{2}}-1}
$$

Where $Q_{\text {ref }}$ is the reference reactive power and $P_{p c c}$ is the active power injection measured at the PCC. We use the dependency between the d-component of the rotor current and the reactive power output described in equation (5) to control the reactive power to the calculated reference value using a PI-controller (see Figure 6).

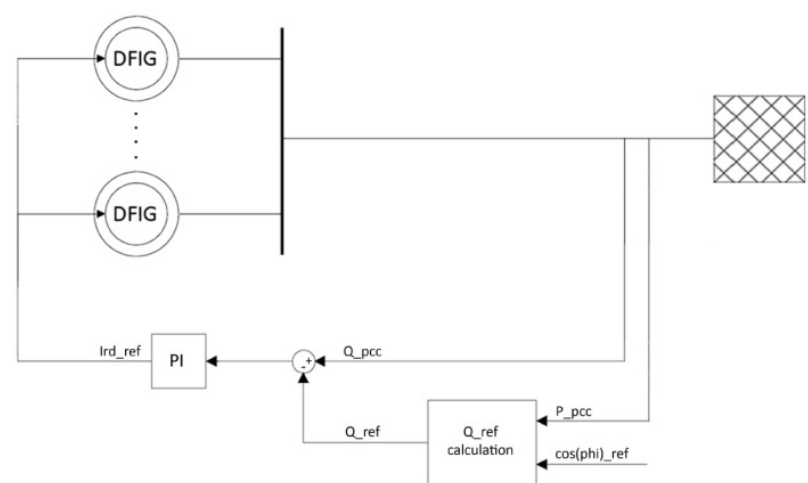

Fig. 6. Reactive Power Control Scheme

Since the reactive power influences the stator voltage, we can no longer guarantee the rated stator voltage. However, the simulation results show, that the stator voltage stay within a $\pm 5 \%$ range of the rated voltage when controlling the wind park to operate at a power factor of $0.96_{\text {ind }}$ (see Figure 7).
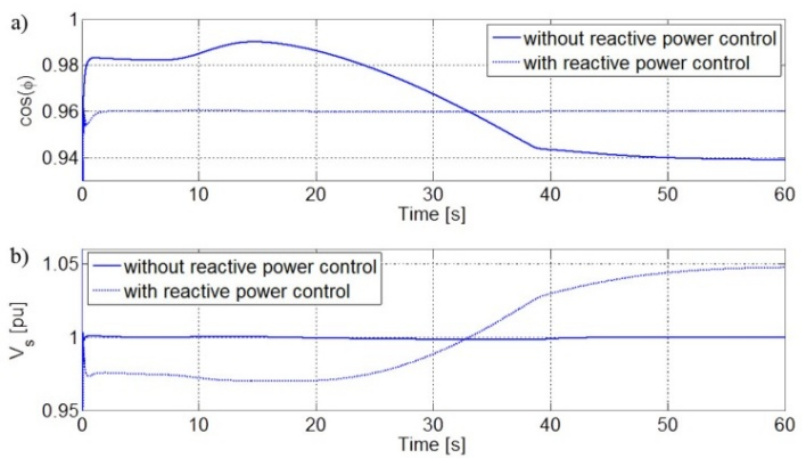

Fig. 7. a) power factor and b) stator voltage

\section{Power Grid Modeling}

\section{A. Structure}

The structure of the considered power grid is shown in Figure 8. It consists of the wind park that is represented by the wind turbine symbol, and the three traditional power plants G1, G2 and G3. The generators are connected by transmission lines that are represented by their impedances Z. In our simulations, generator G1 is operated in load machine mode with a rated power of 7MVA. Generators G2 and G3 generate 3MVA and 4MVA respectively, which means the power system is balanced before the wind park is connected.

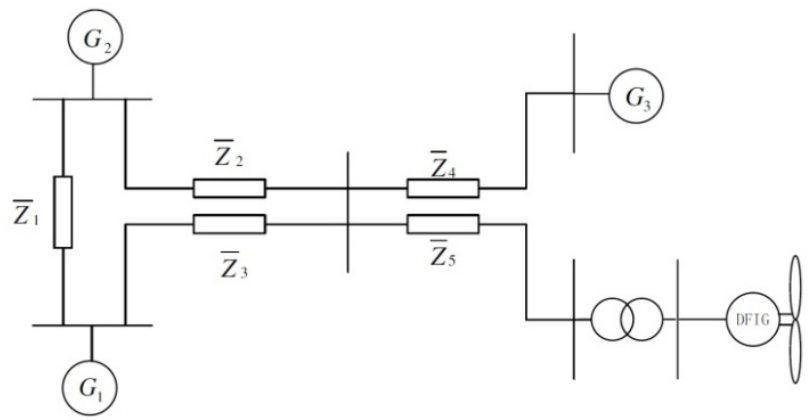

Fig. 8. Structure of the Power System

\section{B. Power Plants}

The power plants in the power system are modeled as synchronous generators. The swing equation describing the balance between electrical and mechanical torque is given as:

$$
\frac{2 \mathrm{H}}{\omega_{s}} \ddot{\delta}+\mathrm{D} \dot{\delta}=P_{m}-P_{e}
$$

With the inertia constant $\mathrm{H}$, damping coefficient $\mathrm{D}$ and generator angle $\delta$. The mechanical power $P_{m}$ equals the rated power of the power plant. The electrical power $P_{e}$ is calculated as

$$
P_{e}=\frac{E^{\prime} V \sin (\delta)}{X_{d}^{\prime}}
$$


with the EMF-voltage $E^{\prime}$, the terminal voltage $V$ and the stator reactance $X_{d}^{\prime}$.

\section{Frequency Control}

Generation and consumption of electrical energy always have to be balanced for the frequency in the power system to remain stable. Therefore, the active power output of the synchronous generators is used to control the frequency in the power grid:

$$
P_{m}=P_{m}^{r e f}-K \delta
$$

with the P-controller $K$ and the control variable $\delta$ indicating frequency deviations.

This approach however fails for wind turbines, since the wind power cannot be influenced. Therefore, the wind park does not participate in frequency control.

\section{Frequency Stability Studies}

\section{A. Transmission Line Length}

First, we connect the wind park to the power grid through different sized transmission lines and analyze the frequency stability in the power grid and at the wind park terminal. The frequency deviations at the wind park and in the power grid can be seen in Fig. 9 .
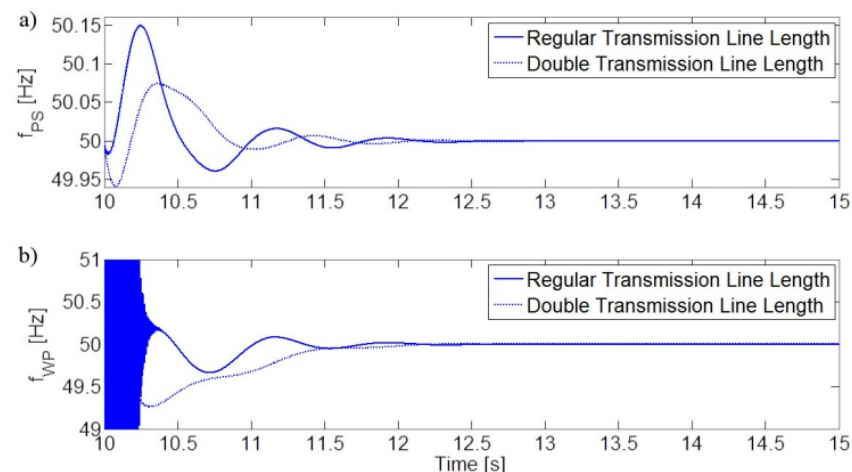

Fig. 9. Frequency deviations after wind park connection a) in the power grid and $b$ ) at the wind park

Our simulations show that the disturbances in the power grid are smaller when the transmission line length is doubled (see Figure 9a). The longer transmission line length damps the effect of the wind park disturbance in the power grid. This effect also holds true in the opposite direction: the impact of the power grid on the wind park is smaller, which is why it takes the wind park longer to settle down at rated frequency (see Figure 9b.). Since the power grid has not as much influence on the wind park when the connection is damped by a long transmission line, the wind park frequency deviates more from the grid frequency. Because of the smaller control ratio of the power grid, the wind park also does not react to the high frequency oscillations that are visible for the regular transmission line length anymore.

From these results we conclude that the grid frequency is less stable if the power plants that are responsible for power control are far away and a disturbance, like a wind park connecting to the power grid, is nearby. Therefore, in grid regions that are close to large wind parks but far away from traditional power plants (for example maritime regions close to wind parks), stability problems can occur.

\section{B. Wind Power Penetration}

We now examine the effect of changing wind energy penetration on the power system. We simulate three differently sized wind parks and observe the impacts in the power grid. The wind parks have 2MVA, 6MVA and 12MVA rated power, which translates into 22.22\%, $46.15 \%$ and $63.16 \%$ of the rated active power respectively. The frequency controllers of generators G2 and G3 are disconnected for this first simulation, which means that the frequency control of the power grid is carried out by G1 alone.

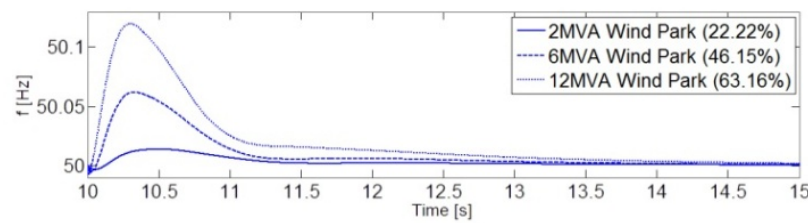

Fig. 10. Frequency deviation after connecting different sized wind parks to the power grid

We measure the mean frequency deviation at the three synchronous generators after connecting the wind park to the power grid. The peak frequency as well as the time it takes to reach stationary state rises with the reference power of the wind park connected to the power grid (see Figure 10). We can therefore conclude that an increase in the penetration of wind energy leads to an increased controlling effort for the traditional power plants.

\section{Frequency Control Depth}

We now examine the impact that the amount of generators that are involved in frequency control have on the frequency stability in the grid. We compare the frequency stability of the power grid when connecting the 12 MVA wind park with all generators controlling the frequency and only G1 controlling the frequency. The difference in the mean frequency at the three synchronous generators after connecting the 12 MVA wind park to the grid can be seen in Figure 11.

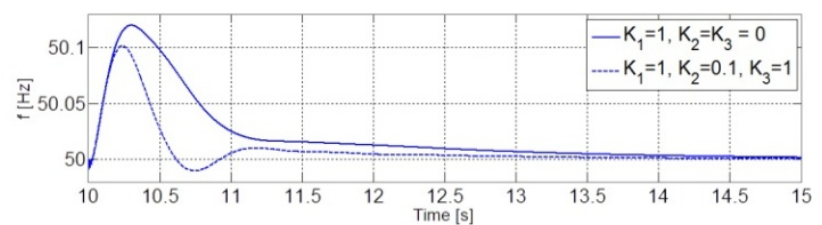

Fig. 11. Frequency deviation after grid disturbance with only one and all generators participating in frequency control

As the simulation shows, the peak frequency is smaller and the stationary state is reached earlier when all generators participate in frequency control. It is therefore important for the power system stability to include as much power plants into the frequency control as possible. 


\section{DFIG Frequency Controller}

The simulations results presented above indicate that frequency stability in power systems can be affected with rising wind power penetration, especially in grid areas close to wind parks. This raises the question, if it is possible to include wind turbines into the frequency control. We recall the frequency controller for the synchronous generator (10) and consider to develop a frequency controller for the wind generator in a similar way.

There are three problems that have to be solved when transferring that approach to the DFIG:

1) Influencing Active Power Output: Since the mechanical power input cannot be influenced, we change the electrical power set point to help balance the frequency deviations in the power grid [3]:

$$
P_{m} \rightarrow P_{e}
$$

The electrical torque is normally controlled to its optimum of $P_{e}^{r e f}$ that guarantees an optimal extraction of electric power from the mechanical power provided by the wind. We give up that optimal power set point in order to help stabilize the power grid. If the frequency rises above its rated value, the electric torque set point is decreased. The electrical power output of the DFIG decreases, which helps to balance the active power surplus in the power system. With the same logic, the electric torque is increased when the grid frequency drops below its rated value. The energy buffer that allows us to vary the active power output short-term is the kinetic energy stored in the rotor. If the electrical torque is set below its optimum, the surplus mechanical torque provided by the wind is used to accelerate the rotor and increase the kinetic energy $E_{\text {kin. }}$. If it is set above the optimum, the power controller taps into the kinetic energy stored in the rotating mass and the rotor slows down.

2) Measuring Deviations in Grid Frequency: the deviation of the angular rotor speed is used as an indicator for grid frequency changes in synchronous generators. This approach fails for induction generators, since the angular speed of the rotor and the frequency of the stator voltages are separated by the generator slip. In [3], the grid frequency is used as the control variable. However, the grid frequency has to be measured in the power grid and fed back to the controller. We therefore propose to use the deviation of the terminal voltage phasor in dq-coordinates as indicator for frequency deviations in the power grid: since the dq-reference frame is rotating with the rated frequency of the power grid, the terminal voltage phasor should stand still in the dq-reference frame when the voltage oscillates with rated frequency. If the voltage frequency changes, the angle of the voltage phasor in dqcoordinates also changes. We therefore define:

$$
\delta_{\text {DFIG }}=\angle \underline{\dot{U}}_{d q}
$$

Since DFIG active power controllers are usually already implemented in dq-coordinates, this variable can easily be obtained inside of the DFIG control-scheme.

3) Managing the Energy Buffer: with this controller, the energy is not lost, but merely transformed and stored. However, the fact that the energy remains inside the system is not enough. We also need to manage the kinetic rotor energy in a way that the rotor speed stays on its optimum course in the long run. We have to feed the surplus energy that we store in the rotor back into the grid, as well as reaccelerate the rotor after kinetic energy was taken out of it. The controller that is modeled until now cannot provide that: when the power system is balanced, the torque set point returns to its optimal set point. However, the kinetic energy surplus or deficit remains in the rotor, since the torque set point would have to exceed or fall behind the optimal point to balance the effect of the controller. To solve this problem, we propose to extend the controller proposed in [3] by a controller that exactly countervails the frequency controller, only with a time delay $\tau$. That way, we can make sure that every time the kinetic energy in the rotor is used as a buffer in short-term, the effect is neutralized in the long run.

With these assumptions, the DFIG frequency controller is given as:

$$
P_{e}=P_{e}^{r e f}-K \delta_{D F I G}\left(1-\frac{1}{1+s \tau}\right)
$$

We can see that:

$$
\lim _{s \rightarrow 0} P_{e}=P_{e}^{r e f}
$$

which shows that the controller does not influence the electric power set point in the long run, but only in short term. This is exactly the behavior we require for our frequency controller.

To test the effect of the controller, we simulate three disturbances in the power grid: we connect the wind park after 10s (Figure 12a), disconnect synchronous generator G3 after 30s (Figure 12b) and reconnect G3 after 50s (Figure 12c). We observe a stabilizing effect of the frequency controller in all three cases. The effect is clearly the strongest when the wind park is connected, because the wind park itself poses the disturbance in this case and therefore is most effective in antagonizing it. But we can also see an effect in controlling grid disturbances: for the first disturbance at 30s, the peek frequency is reduced by $4.62 \%$ and the power system reaches steady state about $25 \%$ earlier.

The influence of the buffer management can be seen in Figure 13 . We can see that without the extension, the 
active power output stays significantly below the optimal course (Figure 13a), while the kinetic energy exceeds the optimal course (Figure 13b). With the extension, active power and kinetic energy only deviate marginally from their optimal courses which reduces the losses that are sustained from stabilizing the frequency significantly. After 150s simulation time, when the transients of all three disturbances are absorbed, the controller losses in respect to generated work add up to only $0.32 \%$.
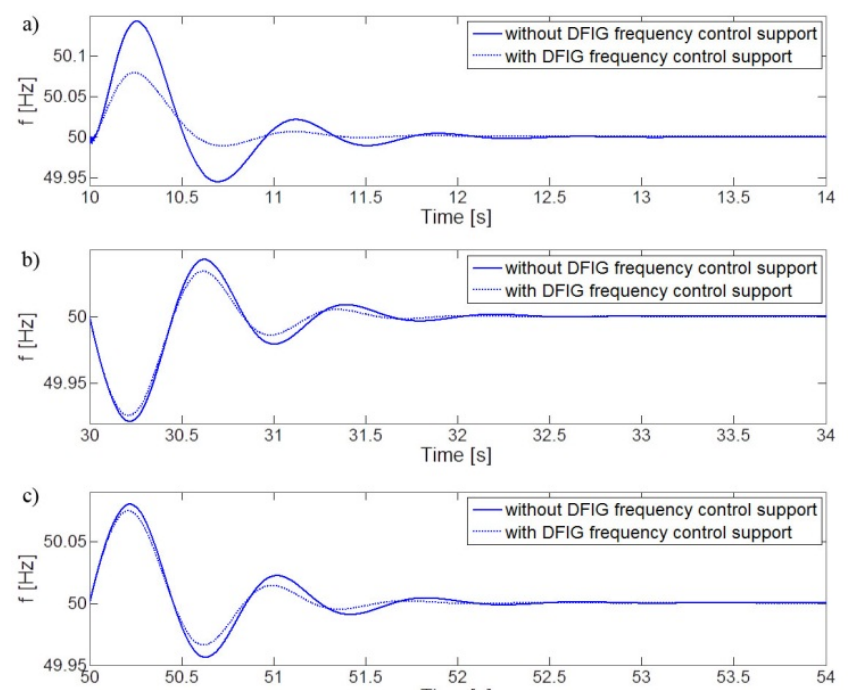

Fig. 12. Frequency responses to a) wind park connection, b) frequency drop and c) frequency rise
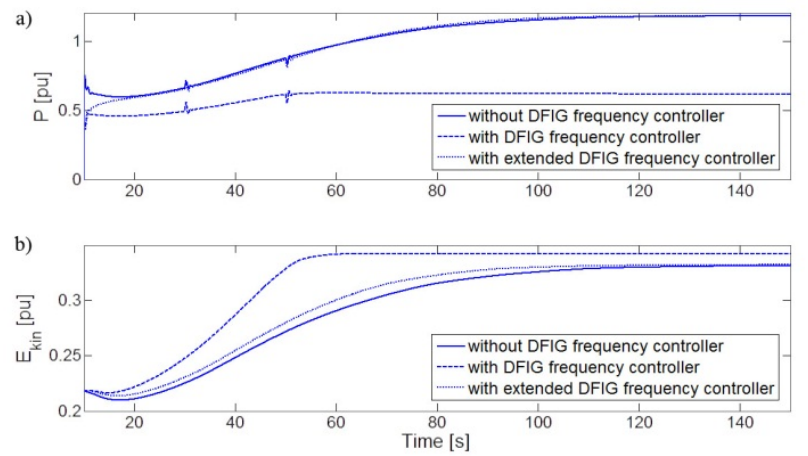

Fig. 13. Course of a) active power and b) kinetic rotor energy

\section{Conclusion}

Our simulations showed that the power grid is more stable near to the generators that are controlling the frequency and less stable near the wind park, that does not participate in frequency control. We were able to show that distances in power grids can be considered as a damper: both positive effects, like control effort, and negative effects, like disturbances are damped by transmission lines. We were also able to show that the power grid gains stability with the amount of generators that are involved in frequency control. We concluded from these results that stability problems in grid regions near wind parks could arise and therefore studied the possibility of including the wind park into the frequency control. Based on a solution presented in [3] that uses the kinetic energy stored inside the generator rotor to vary the active power short-term, we extended this solution with a countervailing, time-delayed controller that balances surpluses or deficits in the kinetic energy of the rotor. We were able to show that this controller stabilizes the power grid with only marginal losses. To the best of our knowledge, a control scheme like this has not been presented in the literature yet. We believe that our approach is a good way to support the short-term frequency stability in power systems and minimize disturbances when connecting wind parks to the power grid.

\section{References}

[1] F. Shewarega, I. Erlich and J. L. Rueda, „Impact of Large Offshore Wind Farms on Power System Transient Stability“, Power Systems Conference and Exposition 2009, Seattle, WA, pp. 1-8.

[2] M. Z. C. Wanik, I. Erlich, A. Mohamed and A. A. Salam, „Influence of Distributed Generations and Renewable Energy Resources Power Plant on Power System Transient Stability“, IEEE International Conference on Power and Energy 2010, Kuala Lumpur, Malaysia, pp. 420-425.

[3] J. Morren, S. de Haan, W. Kling, J. A. Ferreira, "Wind Turbines Emulating Inertia and Supporting Primary Frequency Control“, IEEE Transactions on Power Systems, Vol. 21, No. 1 2006, pp. 433-434.

[4] T. Ackermann, Wind Power in Power Systems, John Wiley \& Sons, (2005).

[5] M. Fujimitsu, T. Komatsu, K. Koyanagi, K. Hu and R.Yokoyama, „Modeling of Doubly-fed Adjustable-Speed Machine for Analytical Studies on Long-term Dynamics of Power System“

[6] Y. Ma, H. Yang, X. Zhou. J. Li and H. Wen, „The Dynamic Modeling of Wind Farms Considering Wake Effects and its Optimal Distribution“, 2009 World NonGridConnected Wind Power and Energy Conference (2009), Nanjing, China, pp. 1-4. 